\title{
Fundamental Constants as Monitors of the Universe
}

Rodger I. Thompson

Department of Astronomy and Steward Observatory, University of Arizona, Tucson, Arizona 85721, USA

E-mail: rit@email.arizona.edu

\begin{abstract}
Astronomical observations have a unique ability to determine the laws of physics at distant times in the universe. They, therefore, have particular relevance in answering the basic question as to whether the laws of physics are invariant with time. The dimesionless fundamental constants, such as the proton to electron mass ratio and the fine structure constant are key elements in the investigation. If they vary with time then the answer is clearly that the laws of physics are not invariant with time and significant new physics must be developed to describe the universe. Limits on their variance, on the other hand, constrains the parameter space available to new physics that requires a variation with time of basic physical law. There are now observational constraints on the time variation of the proton to electron mass ratio $\mu$ at the $10^{-7}$ level. Constraints on the variation of the fine structure constant $\alpha$ are less rigorous $\left(10^{-5}\right)$ but are imposed at higher redshift. The implications of these limits on new cosmologies that require rolling scalar fields has already had its first investigations. Here we address the implications on basic particle physics. The proton to electron mass ratio is obviously dependent on the particle physics parameters that set the mass of the proton and the electron. To first order the ratio is dependent on a combination of the Quantum Chromodynamic scale, the Yukawa couplings, and the Higgs Vacuum Expectation Value. Here that relationship is quantitative defined for the first time. When coupled with previous determinations of the relation of the fine structure constant to the same parameters two constraints exist on the fractional variation of these parameters with time. A third independent constraint involving only the three parameters could set the stage for constraints on their individual fractional variation.
\end{abstract}

keywords: Cosmology, Fundamental Constants, Particle Physics

\section{Introduction}

The considerable evidence for late time acceleration of the expansion of the universe has also accelerated considerations of cosmologies other than the conventional $\Lambda$ CDM cosmology and new physics beyond the standard model. Inherent in many of these alternative cosmologies and new physics is the possibility that fundamental constants such as the proton to electron mass ratio $\mu$ and the fine structure constant $\alpha$ may roll with time. The cosmological implications of such variations in time has previously been discussed in [1], [2] and [3], therefore the emphasis here is on the implications for new physics. Variations of $\mu$ and $\alpha$ requires variations in basic physics parameters such as the Quantum Chromodynamic scale, $\Lambda_{Q C D}$, the Yukawa couplings, $h$ and the Higgs Vacuum Expectation Value $\nu$. The expected time scales for a variation of the constants is on the order of a Hubble time which points to astronomical observations as the natural mode for detecting any change. The implication of a change in $\alpha$ for the parameters has been previously explored in Ref. [4] and [5]. Building on previous work such as e.g. Refs. [6], [7], [8], [4], [5], [9] and [10] a connection between a variance of $\mu$ and the parameters is developed. A limit on the variation of $\mu$ at a redshift of 0.89 establishes the stability of $\mu$ to a part in $10^{7}$ over a time period of a bit more than the last half of the age of the universe. Combined with the limits on (or observation of) a change in 


\begin{tabular}{lllll}
\hline \hline Object & Redshift & $\Delta \mu / \mu$ & $1 \sigma$ error & Ref. \\
\hline J1443+2724 & 4.224 & $-9.5 \times 10^{-6}$ & $\pm 7.6 \times 10^{-6}$ & Ref. [21] \\
Q0347-383 & 3.0249 & $8.2 \times 10^{-6}$ & $\pm 7.4 \times 10^{-6}$ & Ref. [22] \\
Q0528-250 & 2.811 & $3.0 \times 10^{-7}$ & $\pm 3.7 \times 10^{-6}$ & Ref. [23] \\
J1237+0647 & 2.689 & $-5.4 \times 10^{-6}$ & $\pm 7.5 \times 10^{-6}$ & Ref. [24] \\
Q J0643-5041 & 2.659 & $7.3 \times 10^{-6}$ & $\pm 5.9 \times 10^{-6}$ & Ref. [25] \\
Q0405-443 & 2.5974 & $10.1 \times 10^{-6}$ & $\pm 6.2 \times 10^{-6}$ & Ref. [22] \\
Q2348-011 & 2.426 & $-6.8 \times 10^{-6}$ & $\pm 27.8 \times 10^{-6}$ & Ref. [26] \\
He0027-1836 & 2.402 & $-7.6 \times 10^{-6}$ & $\pm 1.0 \times 10^{-5}$ & Ref. [27] \\
J2123-005 & 2.059 & $5.6 \times 10^{-6}$ & $\pm 6.2 \times 10^{-6}$ & Ref. [28] \\
PKS1830-211 & 0.88582 & $-2.9 \times 10^{-8}$ & $\pm 5.7 \times 10^{-8}$ & Ref. [17] \\
B0218+357 & 0.6847 & $-3.5 \times 10^{-7}$ & $\pm 1.2 \times 10^{-7}$ & Ref. [16] \\
\hline \hline
\end{tabular}

Table 1: Current best observational constraints on $\frac{\Delta \mu}{\mu}$

$\alpha$ these observations define the allowed parameter space for deviations from the Standard Model of physics, a subject with few observational constraints. In section 2] the current observational limits on the fractional variations of $\mu$ and $\alpha$ are discussed followed by the development of the relation betwen the fractional variation of $\mu$ and the fractional variations of $\Lambda_{Q C D}, \nu$ and $h$. The previously determined relation of $\alpha$ is introduced to produce a set of two independent equations for the variation of th three parameters and the possiblity of a third independent relationship is discussed.

\section{$2 \mu$ and $\alpha$ Observations}

Astronomical spectroscopic observations that set limits on the variation of $\mu$ and $\alpha$ are generally observations of cold gas in Damped Lyman Alpha clouds (DLAs) that lie along the line of sight to a quasar. This gas produces absorption lines that are generally narrow and whose wavelengths can be established with reasonable accuracy. As its name implies, $\alpha$ measurements are made by observing atomic fine structure transitions in several different elements. Measurements of $\mu$, on the other hand, involve searching for shifts in the spectra of molecular species such as molecular hydrogen Ref. [11]. While the $\alpha$ observations are all at optical wavelengths the $\mu$ measurements include both optical and radio observations. The higher spectral resolution of radio measurements provides very stringent constraints on a variation of $\mu$. The situation with regard to $\alpha$ is a bit murky. There are reports of both temporal and spatial changes in $\alpha$ (Ref. [12], Ref. [13]) at the $10^{-5}$ level which have been a subject of discussion. In contrast recent dedicated VLT with UVES observations (Ref. [14], Ref. [15]) have not been able to confirm these reports.

\section{$2.1 \mu$ measurements}

Observational constraints on $\frac{\Delta \mu}{\mu}$ exist over the redshift range from 0.68 to 4.2 . The most stringent limits come from radio observations of methanol and ammonia absorption lines in two systems with redshifts less than one (Ref. [16], Ref. [17]). These are the limits used here but for completeness Table 1 lists the most stringent $\frac{\Delta \mu}{\mu}$ constraints for the ten objects examined for changes in $\mu$. Fig. 1] shows the two low redshift observations in detail and Fig. 22 shows all of the observations. The radio observations errors are barely visible in Fig. 2 indicating the significantly improved constraints that the radio observations provide. 


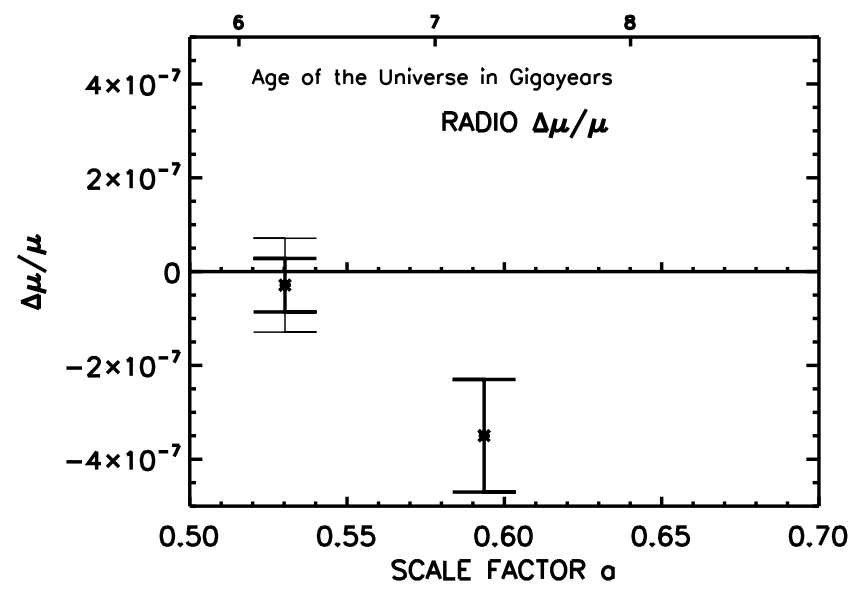

Figure 1: The low redshift radio $\Delta \mu / \mu$ constraints at $z=0.6874$ and $z=0.88582$ plotted versus the scale factor $a=1 /(1+z)$. The error bars are $1 \sigma$, however, an additional larger error bar representing systematic effects is over plotted on the $\mathbf{z}=0.88582(a=0.53)$ constraint. These are the primary constraints utilized in this letter. The age of the universe in gigayears is plotted on the top axis and in Fig. 2.

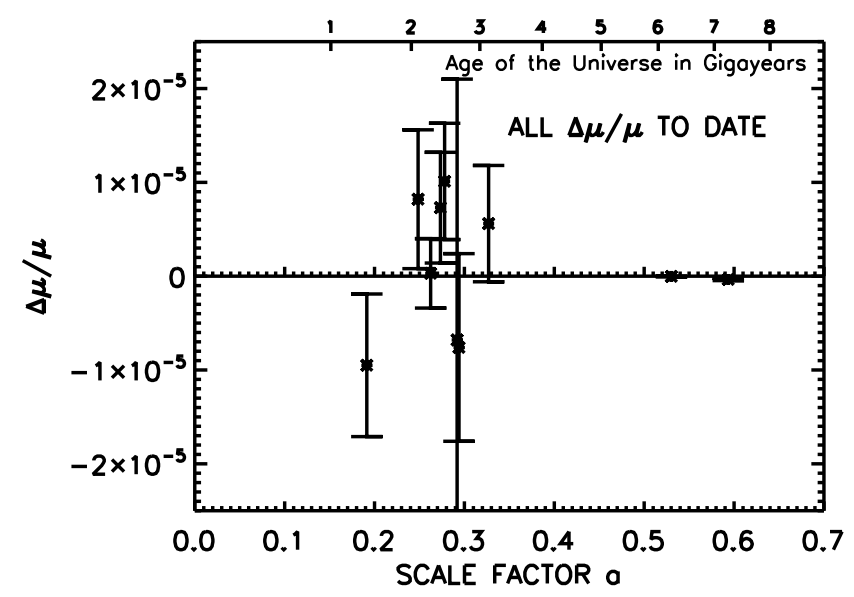

Figure 2: All of the observational constraints on $\Delta \mu / \mu$ from radio $(z<1)$ and optical $(z>1)$ observations plotted versus the scale factor $a=1 /(1+z)$. All constraints are at the $1 \sigma$ level. The low redshift radio constraints are difficult to see at the scale of this plot. 
To date there is no substantiated $(>5 \sigma) \mu$ variation at any redshift. Changes in $\mu$ are detected by changes in molecular spectra Ref. [11] which accounts for the paucity of objects since molecular absorption features are found in only a few extragalactic sources. The PKS1830-211 methanol radio observation Ref. [17] is the most stringent constraint on a change in $\mu$ or at $\frac{\Delta \mu}{\mu}=(-2.9 \pm 5.7) \times 10^{-8}$. This is the statistical limit using all four of the observed methanol absorption lines which includes the $12.179 \mathrm{GHz}$ line. Doubts are expressed in Ref. [17] that this line has a completely common line of sight with the other three lines increasing the uncertainty to $10^{-7}$. The statistical result is retained in Table 1 and Fig. 2 but a $1 \sigma$ uncertainty of $\frac{\Delta \mu}{\mu}=10^{-7}$ is utilized in this analysis and Fig. 11. This new constraint is a primary motivation for exploring the constraints on other fundamental physical parameters.

The new limits on $\frac{\Delta \mu}{\mu}$ are at redshifts less than one, however, this time period is $57 \%$ of the age of a flat universe with $H_{0}=70$ and $\Omega_{m}=0.3$. For comparison the bound on $\frac{\Delta \mu}{\mu}$ at $z=0.88582$ is equivalent to a linear time evolution of less than $\pm 7.88 \times 10^{-18}$ per year as compared to current atomic clock measurements of $\pm 5.8 \times 10^{-16}$ per year Ref. [9]. A linear evolution, however is very unlikely and is just used here as a comparison to the laboratory value.

\section{$2.2 \alpha$ measurements}

It is beyond the scope of this article to thoroughly discuss the current state of $\alpha$ measurements. If the claims of a temporal and spatial variation of $\alpha$ by Ref. [13] are true it would require a major deviation from the standard model. For the purposes of this paper $\alpha$ is assumed to be stable at approximately the $10^{-5}$ level consistent with Ref. [15] and Ref. [14]. This does not affect the main result of the paper which is the connection between $\mu$ and the parameters.

\section{The dependence of $\mu$ on $\Lambda_{Q C D}, \nu$ and $\mathbf{h}_{e}$}

The new stringent constraints on $\frac{\Delta \mu}{\mu}$ provide a strong motivation to determine the equivalent constraints they impose on the fundamental physics parameters that set the proton and electron masses. In the standard model these parameters do not vary but in super symmetry (SUSY) and other new physics theories they can. The constraints on the variance of $\mu$ therefore determine how far the new theories can stray from the standard model. The variation of $\mu$ is simply

$$
\frac{d \mu}{\mu}=\frac{d m_{p}}{m_{p}}-\frac{d m_{e}}{m_{e}}
$$

The proton mass variation involves all three of the fundamental parameters Ref. [4].

$$
\frac{d m_{p}}{m_{p}}=a \frac{d \Lambda_{Q C D}}{\Lambda_{Q C D}}+b\left(\frac{d h_{e}}{h_{e}}+\frac{d \nu}{\nu}\right)
$$

where $a$ and $b$ are constants of order unity and $h_{e}$ is the electron Yukawa coupling. In eqn. 2 the coefficients of the right hand terms have been left to be the general coefficients $a$ and $b$ but in Ref. [4] they have been set to add to one, $a=0.76$ and $b=0.24$. This is because the proton mass is assumed to be proportional to a factor given by $\left(\Lambda_{Q C D}\right)^{a}\left(h_{e} \nu\right)^{b}$. Both $\Lambda_{Q C D}$ and $\nu$ have the units of mass while the Yukawa coupling $h_{e}$ is dimensionless. Since the product must have the dimensions of mass to match the proton mass the powers $a$ and $b$ must add to one. When the logarithmic derivatives are taken on both sides the powers become the coefficients and therefore must add to one as they do in Ref. [4]. If, however, there was another term in the proportionality that had the 
units of mass but did not vary then it would not appear in eqn. 2 and the coefficients would not add to one. For this reason the coefficients are left as the general $a$ and $b$.

By definition the electron mass is $\nu h_{e}$, the product of the Higgs VEV and the electron Yukawa coupling, therefore

$$
\frac{d m_{e}}{m_{e}}=\frac{d h_{e}}{h_{e}}+\frac{d \nu}{\nu}
$$

Combining eqns. 1] 2] and 3 yields

$$
\frac{d \mu}{\mu}=a \frac{d \Lambda_{Q C D}}{\Lambda_{Q C D}}+(b-1)\left(\frac{d h}{h}+\frac{d \nu}{\nu}\right)
$$

using the common assumption that the logarithmic derivatives $\frac{d h_{i}}{h_{i}}$ are the same for all $i$ (Ref. [4]). If $a$ plus $b$ add to one then the much cleaner expression in eqn. 5 ] is true.

$$
\frac{d \mu}{\mu}=a\left(\frac{d \Lambda_{Q C D}}{\Lambda_{Q C D}}-\left(\frac{d h}{h}+\frac{d \nu}{\nu}\right)\right)
$$

Eqn. 4 provides the connection between a change in the dimensionless fundamental constant $\mu$ and a combination of changes in $\Lambda_{Q C D}, h$ and $\nu$. It assumes that the standard model relation between the parameters and the masses of the proton and electron still hold but unlike the standard model they are allowed to vary. The connection is, of course, consistent with the standard model where all of the terms are zero. Note that this is different from recent work by Ref. [18] and Ref. [19] who just considered a variation of the proton mass and restricted its change to only a variation of $\Lambda_{Q C D}$. The observational limit on $\Delta \mu / \mu$ then gives

$$
a \frac{d \Lambda_{Q C D}}{\Lambda_{Q C D}}+(b-1)\left(\frac{d h}{h}+\frac{d \nu}{\nu}\right)<10^{-7}
$$

over the last $57 \%$ of the age of the universe. The previously derived connection for $\alpha$ (Ref. [4], Ref. [5]) is

$$
R \frac{\Delta \alpha}{\alpha}=\frac{\Delta \Lambda_{Q C D}}{\Lambda_{Q C D}}-\frac{2}{27}\left(3 \frac{\Delta \nu}{\nu}+\frac{\Delta h_{c}}{h_{c}}+\frac{\Delta h_{b}}{h_{b}}+\frac{\Delta h_{t}}{h_{t}}\right)
$$

where $\mathrm{R}$ is a model dependent scalar. Under the assumption that $\frac{\Delta h_{i}}{h_{i}}=\frac{\Delta h}{h}$ it becomes

$$
\frac{\Delta \alpha}{\alpha}=\frac{1}{R}\left[\frac{\Delta \Lambda_{Q C D}}{\Lambda_{Q C D}}-\frac{2}{9}\left(\frac{\Delta \nu}{\nu}+\frac{\Delta h}{h}\right)\right]<10^{-5}
$$

Equations 6 and 8 relate fractional changes in $\mu$ and $\alpha$ to fractional changes in $\Lambda_{Q C D}, \nu$ and $h$. The far right hand term in each of the relations represents the current observational constraints on $\mu$ and $\alpha$.

Equations 6 and 8 are two equations in the three unknowns $\Delta \Lambda_{Q C D} / \Lambda_{Q C D}, \Delta \nu / \nu$, and $\Delta h / h$. As such they allow the ellimination of one of the variables but the resultant is still the combination of two of the unknowns. A third independent equation would allow the separation of the unknowns into three separate constraints on the individual parameters. A possibility is the gyromagnetic ratio $g$ which can be expressed in a Taylor series expansion in $\alpha$. Hyperfine splitting, such as observed in the $21 \mathrm{~cm}$ line is dependent on $g$ along with $\alpha$ and $\mu$ which could provide an observational channel for the third equation. 


\section{Conclusions}

Fundamental constants such as $\mu$ and $\alpha$ are sensitive monitors of possible changes in time of the basic laws of physics. Both involve complex physics that have many components and interrelationships such that the variance of any of the parameters can not be considered in isolation from the rest. The fundamental constants, however, are pure numbers and changes in those numbers are unambiguous and measurable. If the standard model relationships between the fundamental constants and the dimensionfull parameters are maintained fractional changes in the constants establish quantitative connections to fractional changes in the parameters. This work provides the connection between fractional changes in $\mu$ and a combination of fractional changes in the quantum chromodynamic scale, the Higgs vacuum expectation energy and the Yukawa couplings. This connection has model dependent parameters as does the previously established connection with fractional changes in $\alpha$. A third possible constraint involves the gyromagnetic ratio $g$. Three indepedent equations raises the possibility of constraining the three parameters separately. To date no confirmed change with time has been found for either $\mu$ or $\alpha$ on astronomical time scales. These observational limits then provide quantitative limits on the allowed variance of the combination of parameters but not on the individual parameters themselves. The observational limits are consistent with the standard model and provide limits on the allowed deviations from that model.

\section{Acknowledgements}

The author thanks Jullian Berengut for enlightening discussions on fundamental constant variation versus parameter variation and Keith Olive for discussions clarifying the dimensional argument of why the coefficients in equation 11 of Ref. [4] should add to one. C. J. A. P. Martins is thanked for useful discussions in preparing this article. The author also benefited from discussions with $D$. Psaltis, D. Arnett, A. Coc and P. Pinto.

\section{References}

[1] Calebrase, E. Menegoni, E. Martins, C.J.A.P., Melchiorri, A. and Rocha, G., Phys. Rev. D, 84, 02358 (2011)

[2] Thompson, R.I., MNRAS Letters, 422, L67 (2012)

[3] Thompson, R.I., Martins, C.J.A.P. and Vielzeuf, P.E., MNRAS, 428, 2232 (2013)

[4] A. Coc, N. J. Nunes, K. A. Olive, J-P Uzan and E. Vangioni, Phys. Rev. D 76, 023511 (2007)

[5] Luo, F., Olive, K.A., \& Uzan, J-P, Phys. Rev. D, 84, 096004 (2011)

[6] Campbell, B.A. and Olive, K.A., Phys. Lett. B, 345, 429 (1995)

[7] Langacker, P., Segre, G. and Strassler, M.J., Phys. Lett. B, 528, 121 (2002)

[8] Damour, T., Astr. and Spa. Sci., 283, 445 (2003)

[9] Ferreira, M.C., Juliao, M.D., Martins, C.J.A.P. and Monteiro, A.M.R.V.L., Phys. Rev. D, 86, 125025 (2012) 
[10] Ferreira, M.C., Frigola, O., Martins,C.J.A.P., Monteiro, A.M.R.V.L, and Sola, J., Phys. Rev. D, 89, 083011 (2014)

[11] Thompson, R.I., Astrophys. Lett., 15, 3 (1975)

[12] Webb, J.K., King, Murphy, M.T., Flambaum, V.V., Dzuba, V.A., Barrow, J.D., Churchill, C.W., Prochaska, J.X. \& Wolfe, A.M., PRL, 87, 091301-1-4 (2001)

[13] Webb, J.K., J.A., Murphy, M.T., Flambaum, V.V., Carswell, R.F., \& Bainbridge, M.B., PRL, 107, 191101-1-5 (2011)

[14] Evans, T.M. et al., MNRAS, 445, 128 (2014)

[15] Molaro, P. et al., A\&A, 555, A68 (2013)

[16] Kanekar, N., Ap.J.L., 728, L12 (2011)

[17] Kanekar, N. et al., MNRAS, 448, L104 (2015)

[18] Calmet, X and Keller, M., arXiv:1410.2765v2 [gr-qc] (2014)

[19] Fritzsch, H. and Sola, J. arXiv:1502.0141v1 [gr-qc] (2015)

[20] Vielzeuf, P. and Martins, C.J.A.P., Mem. Soc. Astro. Ital., 85, 155 (2014)

[21] Bagdonaite, J., Ubachs. W., Murphy, M. T., and Whitmore, J. B., Phys. Rev. Lett., 114, 071301 (2015)

[22] King, J. A., Webb, J. K., Murphy, M. T. \& Carswell, R. F., PRL, 101, 251304 (2009)

[23] King, J. A., Webb, J. K., Murphy, M., Ubachs, W, \& Webb, J., MNRAS, 417, 3010 (2011)

[24] Dapra, M. ,Bagdonaite, J., Murphy, M.T., Ubachs, W., arXiv:1508.07419v1, [astro-ph.CO] (2015)

[25] Rahmani, H., Vasquez, D.A., Srianand, R., Petitjean, P., Noterdaeme, P. and Ledoux, C., Mem. S.A.It,85, $84(2014)$

[26] Bagdonaite, J., Murphy, M. T. , Kaper, L. and Ubachs, W., MNRAS, 421, 419 (2012)

[27] Rahmani et al. 2013, MNRAS, 435, 861 (2012)

[28] Malec, A.L. et al., MNRAS, 403, 1541 (2010) 